\title{
Computed Tomography- Guided Bone Marrow Aspiration and Trephine (Core) Biopsy for Hematologists and Oncologists
}

\author{
Anwarul Islam* \\ Clinical Associate Professor of Medicine, Division of Hematology/Oncology, Department of Medicine, Buffalo General Hospital, \\ USA
}

*Corresponding author: Anwarul Islam, Clinical Associate Professor of Medicine, Division of Hematology/Oncology, Department of Medicine, Buffalo General Hospital, USA

ARTICLE INFO

Received: 幽 March 16, 2020

Published: 絊 March 31, 2020

Citation: Anwarul Islam. Computed Tomography- Guided Bone Marrow Aspiration and Trephine (Core) Biopsy for Hematologists and Oncologists. Biomed J Sci \& Tech Res 26(5)-2020. BJSTR. MS.ID.004409.

Keywords: CT Guidance; Bone Marrow Aspiration; Bone Marrow Trephine Biopsy; Bone Marrow Core Biopsy

\begin{abstract}
Computed tomography (CT) with or without fluoroscopy has evolved as a very useful tool in medical science and is used as an instrument for sampling a solid organ tissue (biopsy), body fluid, or a tumor mass suspected of malignancy. In this system the aspiration and biopsy needle can be visualized by the physician practitioner on a CT scan and help assist in the accurate localization of the target tissue. Because of the problems associated with accurately locating the posterior iliac crest with surface anatomic landmarks and conventional palpation (considered as a blind method) we have used the technique of CT-guided bone marrow aspiration and bone marrow trephine (core) biopsy in patients diagnosed with various hematological malignancies. We found the technique is extremely useful in accurately locating the posterior iliac crest particularly in obese patients. This procedure facilitates an adequate aspiration of a marrow suspension (liquid marrow) and extraction of an excellent core sample each time and is more surgically precise than when access to the target bone (iliac crest) is based only on palpation of external landmarks (blind method).
\end{abstract}

\section{Short Communication}

Bone marrow aspiration and trephine (solid core) biopsy is a crucial tool in the investigation, diagnosis and management of patients with many types of hematological and non-hematological malignant conditions [1-5]. Both procedures are currently used in tandem by hematologists and oncologists because each one offers specific and important diagnostic information [6-9]. However, even in the hands of a skilled professional using the appropriate type of needle, the site (in most cases, the posterior iliac crest) can be missed or not hit at the center of the posterior ilium resulting in an inadequate, poor or unsatisfactory bone marrow aspiration and trephine (solid core) biopsy specimen. In order to efficiently and precisely locate the posterior iliac crest and to obtain sufficient aspirate sample and an adequate length of a core biopsy specimen, we have used CT guidance without fluoroscopy to accurately define and locate the posterior iliac crest prior to obtaining a bone marrow sample.

\section{Materials and Methods}

After obtaining informed consent a CT guided bone marrow aspiration and trephine (solid core) biopsy was obtained from patients diagnosed with various hematological conditions such as leukemia, multiple myeloma, myelodysplastic syndrome, thrombocytopenia, non-Hodgkin's lymphoma and nonhematological malignant conditions such as carcinoma of the breast and prostate with bone metastasis. In most cases the patients were placed in a right or left lateral decubitus position, with the knees drawn up and back comfortably flexed (Figure 1) or in the prone position (Figure 2) in the CT scanner. A grid was placed over the posterior iliac region (Figures $1 \& 2$ ) and the desired entry point of the needle was identified on the grid under CT visualization. The patient was then partly pulled back out of the CT scanner, the grid was removed, and the desired entry point of the needle was marked on the patient's body with an indelible marker. The operative site 
was prepared with the use of sterile technique and the patient was draped. Following local anesthesia (skin, subcutaneous tissue and periosteum) a 15-gauge aspiration needle [10] was introduced through the skin at the earlier marked location and advanced slowly pointing toward the anterior superior iliac spine.

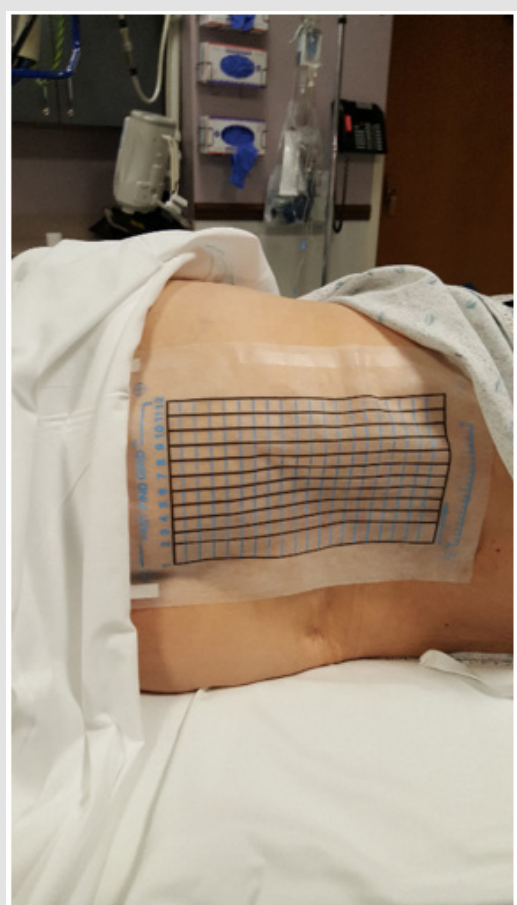

Figure 1: A patient placed in a left lateral decubitus position with the knees drawn up and back comfortably flexed. The grid which helps in selecting the entry site and marking the point of entry of the needle is in place.

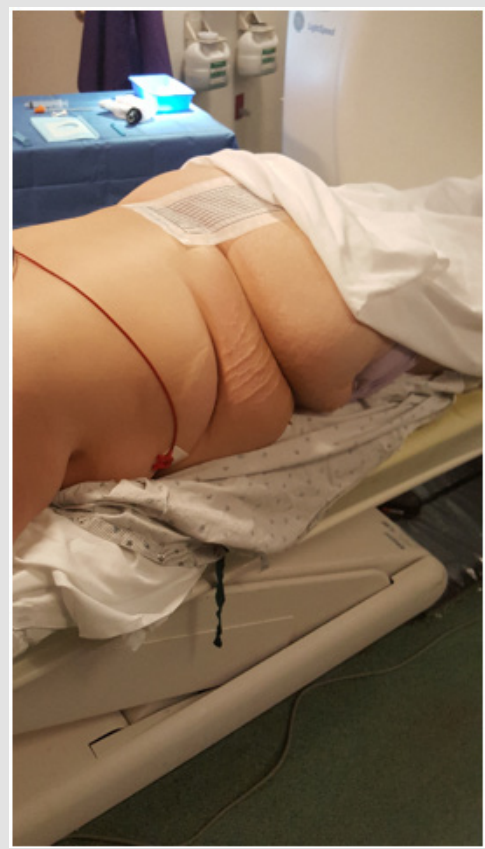

Figure 2: A patient placed in a prone position on the CT scanner. The grid which helps in selecting the entry site and marking the point of entry of the needle is in place.
Once the needle was stabilized (locked) in the bone the patient was moved back into the scanner to visualize whether the tip of the needle was in the correct position. Although such verification is not always necessary, we utilized this procedure as a precautionary measure. Once this was affirmed the patient was again moved out of the scanner and a routine bone marrow aspiration was performed using a standard bone marrow aspiration needle [10]. Following bone marrow aspiration, a small incision was made over the marked area with a surgical scalpel to allow for the passage of the standard (11 gauge) bone marrow core biopsy needle [11]. The biopsy needle with the stilette in place was then advanced slowly through the incision, pointing towards the anterior superior iliac spine and when the posterior iliac crest was reached it was then penetrated by gentle rotary motions of the needle. Once the cortex was penetrated the stilette was then removed with one hand while holding the needle in place with the other. The biopsy needle was then advanced with slow, steady and controlled clockwisecounterclockwise rotary motions until an adequate depth (about 20-25 mm) was reached (Figure 3).

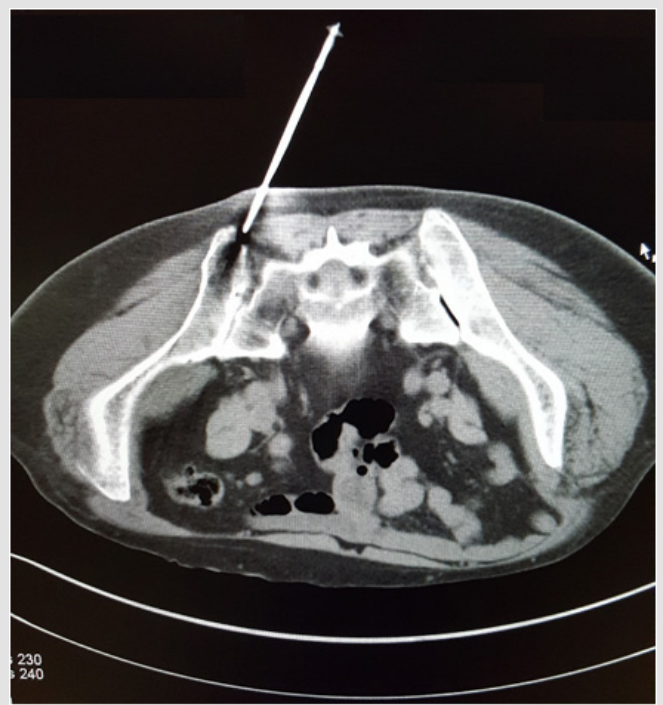

Figure 3: A CT image shows the tip of an 11-gauge Islam bone marrow biopsy needle accurately placed over the posterior ilium near the posterior iliac crest before being advanced into the marrow cavity.

The biopsy needle was then rotated completely several times about its long axis to sever all trabecular connections at its base and break the core sample loose from the surrounding spongy bone and was then slowly withdrawn with a straight pull and gentle alternating rotary motions. No rocking, sculling or change in the direction of the tip of the needle was necessary. Once the needle was withdrawn, the insertion aid was attached to the distal cutting end of the needle. The biopsy specimen was then pushed out of the needle with the pusher that was introduced through the opposite side of the insertion aid. In most patients the procedure was performed under local anesthesia ( 1 or $2 \%$ lidocaine). However, some patients did receive conscious sedation with midazolam 
or a combination of midazolam and fentanyl. In each case a bone marrow aspiration with a 15 gauge needle was performed first prior to bone marrow trephine (core) biopsy with a separate larger 11 gauge biopsy needle because of the advantage this two needle technique offers when both bone marrow aspiration and core biopsy is performed with the same core biopsy needle (one needle technique) [12]. Once the biopsy procedure was complete, the edges of the wound were pressed together with an adhesive tape. A gauze dressing was applied, and the patient was instructed to lie flat on his/her back for 10-15 minutes or longer if the patient had a low platelet count, was on anticoagulation or was taking aspirin.

\section{Results}

A satisfactory (over $15 \mathrm{~mm}$ or longer) bone marrow trephine (core) biopsy specimen (Figure 4) was obtained from each patient. There never was any loss of a core sample as the biopsy needle used contained an internal core retention device [11]. There were neither tangential specimens (i.e., containing mostly bone and subcortical fat tissue) nor any unsatisfactory ones (i.e., small, less than $5 \mathrm{~mm}$ in length or crushed biopsies with little or no marrow tissue). We found the technique was straightforward, easy to understand and use, and very efficacious in precisely locating the posterior ilium and obtaining excellent specimens. The patients tolerated the procedure well.

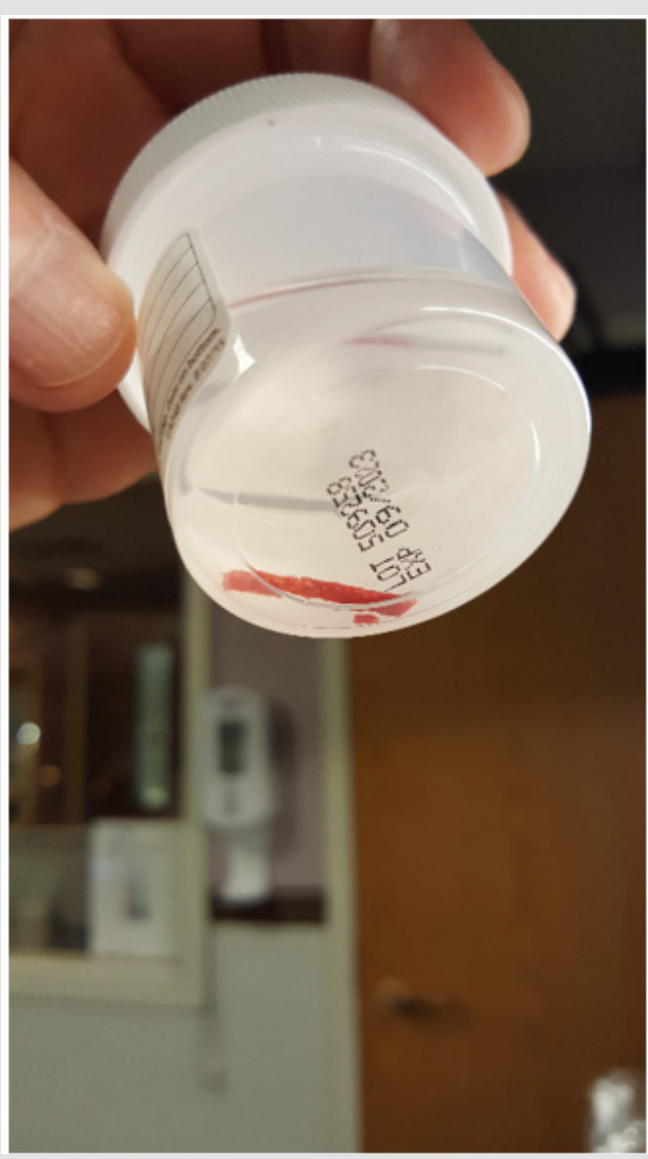

Figure 4: A fresh long core biopsy (over $20 \mathrm{~mm}$ in length) obtained from a patient using a CT guidance.

\section{Discussion}

Conventionally, hematologists and oncologists rely on external anatomical landmarks and palpation to identify and locate the posterior iliac crest to obtain bone marrow aspirate and trephine biopsy specimens. This is known as the blind approach and quite often it proves to be unsatisfactory as the posterior iliac crest is not contacted (missed). We illustrate that the technique of bone marrow aspiration and trephine biopsy from the posterior iliac crest can be vastly improved when it is performed under CT guidance. CT guidance for bone marrow aspiration and biopsy offers multiple advantages over the conventional approach. With this technique the posterior iliac crest can be easily located, and the needle can be directed at a correct angle toward the posterior iliac crest and most importantly the needle can be visualized entering the bone. The success rate of acquiring a satisfactory bone marrow trephine (solid core) biopsy sample is contingent on both the type and make of biopsy needle chosen [13] as well as the skill and knowledge of the professional using it [14]. Much also depends on precisely locating the target site. In this case, it is crucial to be able to locate the posterior iliac crest, as this region is currently preferred site both bone marrow aspiration as well as trephine biopsy). In most situations, hematologists, oncologists and nurse practitioners rely on external anatomical landmarks (blind approach) to locate the posterior iliac crest in order to perform this important diagnostic procedure.

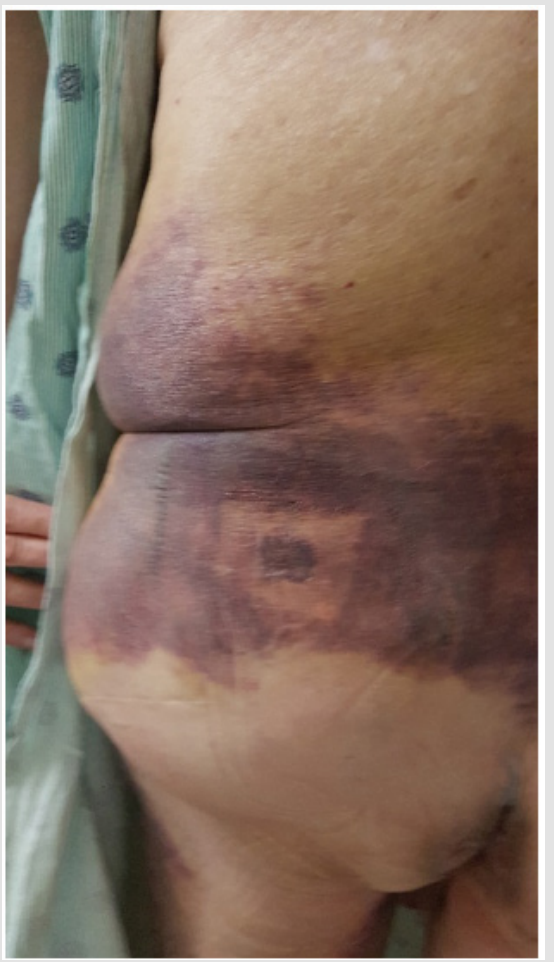

Figure 5: A photograph showing massive extravasation and hematoma formation at the site of the left posterior ilium following a bone marrow aspiration and a biopsy procedure in an elderly patient whose platelet count was normal and was not on aspirin or any anticoagulation. 
Unfortunately, the blind approach method is very liable to miss the center of the bone where the needle should make contact. If the needle misses the center of the bone, it is much less likely to obtain a satisfactory long core biopsy containing trabecular bone. Also, in such cases, marrow tissue may be missed, resulting in an inadequate and unsatisfactory trephine biopsy specimen. Moreover, if the needle does not hit the bone at the correct angle this may result in a tangential biopsy which contains mostly bone and very little of the marrow tissue which is required to make a proper diagnosis. Furthermore, if the needle veers too much to the right, left, or any other direction, or misses posterior iliac crest completely, that can cause serious complications, including damage to the sciatic nerve or a massive extravasation and thigh and buttock hematoma (Figure 5). These complications, although rare, but have been reported $[15,16]$. CT guidance greatly reduces the chance of user error and enables the safe passage of the needle into the desired marrow-rich intramedullary site for ample bone marrow aspiration, resulting in a decent length of a core biopsy specimen while simultaneously avoiding damage to important surrounding structures.

\section{References}

1. Mc Farland W, Dameshek W (1958) Biopsy of bone marrow with the Vim-Silverman needle. JAMA 166(12): 1464-1466.

2. Ellman L (1976) Bone marrow biopsy in the evaluation of lymphoma, carcinoma and granulomatous disorders. Am J Med 60(1): 1-7.

3. Burke JS (1978) The value of the bone marrow biopsy in the diagnosis of hairy cell leukaemia. Am J Clin Pathol 70(6): 876-884.

4. Brunning RD, Bloomfield CD, Mc Kenna RW (1975) Bilateral trephine bone marrow biopsies in lymphoma and other neoplastic disease. Ann Int Med 82(3): 365-366.

ISSN: 2574-1241

DOI: $10.26717 / B J S T R .2020 .26 .004409$

Anwarul Islam. Biomed J Sci \& Tech Res

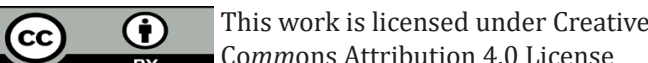

Submission Link: https://biomedres.us/submit-manuscript.php
5. Parapia LA (2007) Trepanning or trephines: a history of bone marrow biopsy. Br J Haematol 139(1): 14-19.

6. Brynes RK, Mc Kenna RW, Sundberg RD (1978) Bone marrow aspiration and trephine biopsy an approach to a through study. Am J Clin pathol 70(5): 753-759.

7. Islam A (1996) Indications for and value of bone marrow trephine biopsy in haematological disorders (invited annotation). Haematology 1(2): 167-172.

8. Moid F, De Palma L (2005) Comparison of relative value of bone marrow aspirates and bone marrow trephine biopsies in the diagnosis of solid tumor metastasis and Hodgkin's lymphoma - Institutional experience and literature review. Arch Pathol Lab Med 129(4): 497-501.

9. Chandra S, Chandra H (2011) Comparison of bone marrow aspirate cytology, touch imprint cytology and trephine biopsy for bone marrow evaluation. Hematology Reports 3(3): e22.

10. Islam A (1983) A new bone marrow aspiration needle to overcome the sampling errors inherent in the technique of bone marrow aspiration. Journal of Clinical Pathology 36(8): 954-958.

11. Islam A (2019) A New Single-Use Bone Marrow Biopsy Needle With Core Retention Design. Biomed J Sci \& Tech Res 19(3): 14293-14299.

12. Islam A (2007) Bone marrow aspiration before bone marrow biopsy using the same bone marrow biopsy needle: A good or bad practice? J Clin Pathol 60(2): 212-215.

13. Jacobs $P$ (1995) Choice of needle for bone marrow trephine biopsies. Hematology reviews 9: 163-168.

14. Rudzki Z, Partyla T, Okon K, Stachura J (2005) Adequacy of trephine bone marrow biopsies: the doctor and the patient make a difference. Pol J Pathology 56(4): 187-195.

15. Bain BJ (2003) Bone marrow biopsy morbidity and mortality. Br J Haematol 121(6): 949-951.

16. Sarigianni M, Vlachaki E, Chissan S, Klonizakis F, Vetsiou E, et al. (2011) Haematoma caused by bone marrow aspiration and trephine biopsy. Hematology Reports 3(3): e25.

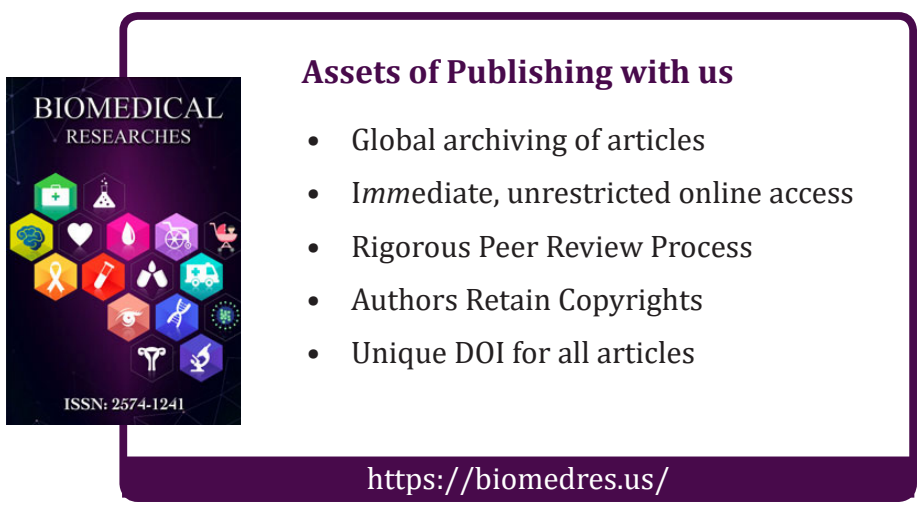

